\title{
On Global Trajectory Tracking Control for an Omnidirectional Mobile Robot with a Displaced Center of Mass
}

\section{A.S. Andreev, O. A. Peregudova}

This paper addresses the trajectory tracking control design of an omnidirectional mobile robot with a center of mass displaced from the geometrical center of the robot platform. Due to the high maneuverability provided by omniwheels, such robots are widely used in industry to transport loads in narrow spaces. As a rule, the center of mass of the load does not coincide with the geometric center of the robot platform. This makes the trajectory tracking control problem of a robot with a displaced center of mass relevant. In this paper, two controllers are constructed that solve the problem of global trajectory tracking control of the robot. The controllers are designed based on the Lyapunov function method. The main difficulty in applying the Lyapunov function method for the trajectory tracking control problem of the robot is that the time derivative of the Lyapunov function is not definite negative, but only semidefinite negative. Moreover, the LaSalle invariance principle is not applicable in this case since the closed-loop system is a nonautonomous system of differential equations. In this paper, it is shown that the quasi-invariance principle for nonautonomous systems of differential equations is much convenient for the asymptotic stability analysis of the closed-loop system. Firstly, we construct an unbounded state feedback controller such as proportional-derivative term plus feedforward. As a result, the global uniform asymptotic stability property of the origin of the closed-loop system has been proved. Secondly, we find that, if the damping forces of the robot are large enough, then the saturated position output feedback controller solves the global trajectory tracking control problem without velocity measurements. The effectiveness of the proposed controllers has been verified through simulation tests. Namely, a comparative analysis of the bounded controller obtained and the well-known "PD+" like control scheme is carried out. It is shown that the approach proposed in this paper saves energy for control inputs.

Received September 17, 2019

Accepted February 11, 2020

The work was supported by the Russian Foundation for Basic Research under Grant [18-01-00702].

\author{
Aleksandr S. Andreev \\ asa5208@mail.ru \\ Olga A. Peregudova \\ peregudovaoa@gmail.com \\ Ulyanovsk State University \\ ul. Lva Tolstogo 42, Ulyanovsk, 432000 Russia
}

RUSSIAN JOURNAL OF NONLINEAR DYNAMICS, 2020, 16(1), 115-131 — \#) 
Besides, a comparative analysis of the bounded controller and its analogue constructed earlier in a cylindrical phase space is carried out. It is shown that the controller provides lower values for the root mean square error of the position and velocity of the closed-loop system.

Keywords: omnidirectional mobile robot, displaced mass center, global trajectory tracking control, output position feedback, Lyapunov function method

\section{Introduction}

Omnidirectional mobile robots known for their maneuverability are used in many areas of the human activity [22]. The most common are the models of three or four-wheeled mobile robots. Many different approaches in the trajectory tracking control design for such models of omnidirectional mobile robots have been proposed in the literature. In [14, 17], kinematical models of three-wheeled mobile robots were considered and position and trajectory tracking control problems were solved taking into account the actuator saturation. In [1], the dynamics of a four-wheeled omnidirectional robot was considered and an algorithm of computing the controller for program motion implementation was proposed. Algorithm for trajectory generation for a four-wheeled omnirobot was proposed in [23]. In [15], the experimental tuning of the proportional integral differential (PID) controller was considered to track the trajectory of a four-wheeled omnimobile robot.

The equations of motion of an omnidirectional mobile robot with three orthogonal universal wheels have been derived by many researchers (see, for example, $[10,18,20,30]$ ). In an orthogonal universal wheel the rollers are aligned around its rim in such a way that the wheel can roll orthogonally to its driving direction [9]. In [18], a trajectory linearization control method with proportional integral (PI) terms was used to achieve an exponential stability of the closed-loop system. A kinematic control method based on feedback linearization was proposed in [13] in order to achieve simultaneous tracking and stabilization. The computed torque method with proportional differential (PD) terms was applied by [28] to solve the trajectory tracking control problem of the robot.

An adaptive backstepping based controller was proposed in [12] for trajectory tracking and stabilization of an omnidirectional wheeled mobile robot with parameter variations and uncertainties. In [11], the sliding mode tracking control design was proposed. In [3, 4] both the position stabilization and trajectory tracking control problems were solved using Lyapunov function method. In [24], the passivity-based controller based on "PD+" approach was proposed to solve the global trajectory tracking control problem using only position measurements. Many researchers applied the model-predictive control design to solve the trajectory tracking problem $[8,26,29]$.

Note that in all the above-mentioned papers, a dynamic model was considered wherein the mass center of the robot coincides with the geometric center of the platform. In practice, such a requirement is difficult to achieve due to the operating conditions of the robot. For example, such robots are used to move various loads in narrow spaces. Therefore, the study of the dynamics and the trajectory tracking control of the robot with a displaced mass center are problems of current interest. In [16], the kinematic model of an omniwheeled spherical robot was considered taking into account the displacement of the center of mass inside the moving platform.

In this paper, we address the global trajectory tracking control problem of an omnidirectional mobile robot with a displaced mass center. The equations of motion for such a robot 
model were obtained in [19]. To date, a nonlinear PI controller has been proposed in [5] to solve the position stabilization problem. The problem of trajectory tracking control design for such a robot model was considered in the previous papers of the authors $[6,7]$ wherein the output position feedback controllers were constructed taking into account such features as periodicity of the model equations in the angular coordinate of the robot as well as the presence of delay in the control feedback.

The main contributions of this paper are as follows. Firstly, we propose a novel controller inspired by the so-called PD+ approach to solve the global trajectory tracking control problem for the nonlinear model of an omnidirectional mobile robot with a displaced mass center. Secondly, we propose a stability analysis that provides the global uniform asymptotic stability property of the closed-loop system using a Lyapunov function with a semidefinite time derivative on the basis of the theory of limiting equations. Thirdly, we propose a bounded position output feedback controller which solves the global trajectory tracking control problem without velocity measurements.

Throughout this paper the following mathematical notations are used. $E \in \mathbb{R}^{n \times n}$ is the identity matrix. Superscript $T$ denotes a transpose operation. Symbol $\|\cdot\|$ denotes the Euclidean vector norm.

\section{Formulation of the Problem}

Consider a dynamical model of a mobile robot (see, Fig. 1) with three omnidirectional wheels and a displaced center of mass, represented in [19]. We use the following notation and assumptions. The world coordinate frame $O X Y Z$ is fixed on the ground plane $O X Y$. $O Z$ is the axis up. Let $C$ be the center of an equilateral triangle $C_{1} C_{2} C_{3}$, at the vertices of which the centers of the robot wheels are situated. $C x_{1} y_{1} z_{1}$ is a body coordinate frame fixed at the moving platform of the robot. The axis $C x_{1}$ is parallel to the axis of rotation of the first wheel; the axis $C z_{1}$ is vertical; the axes $C y_{1}, C z_{1}$ and $C x_{1}$ form a right-handed coordinate frame. The planes $O X Y$ and $C x_{1} y_{1}$ are parallel.

Neglecting the mass and dimensions of the rollers, consider the robot model as a mechanical system of four absolutely rigid bodies, the position of which is determined by six independent parameters $x, y, \psi, \varphi_{1}, \varphi_{2}$ and $\varphi_{3}$, where $x$ and $y$ denote the platform center location in the world frame $O X Y Z ; \psi$ is the platform orientation angle measured from the axis $O X ; \varphi_{j}$ is the

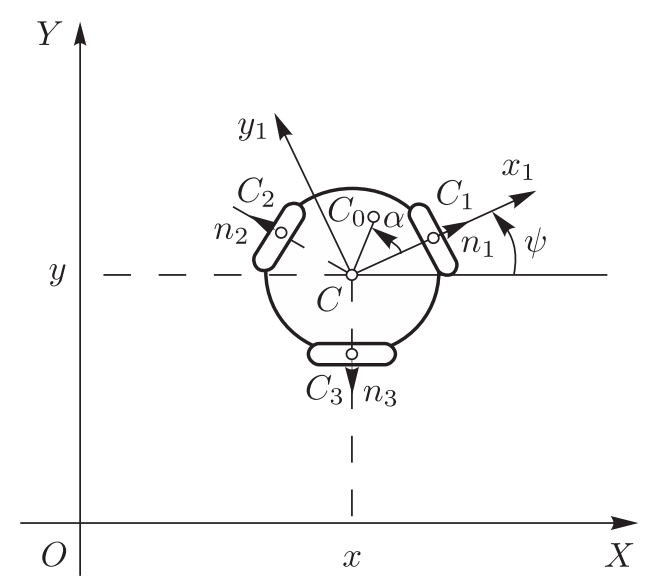

Fig. 1. Robot's model. 
angle of rotation of the $j$ th wheel relative to its axis of rotation $(j=1,2,3)$. Denote the positive direction (counterclockwise) of the $i$ th wheel rotation by the vector $n_{i}, i=1,2,3$. The mass center of the system $C_{0}$ is shifted relative to the center $C$ of the platform by distance $d$. The angle between the axis $C x_{1}$ and the vector $C C_{0}$ is denoted by $\alpha$. Denote by $r$ the radius of the wheels. The distances from the center of the platform to the center $C_{j}(j=1,2,3)$ of each wheel are equal to $a$. Denote the masses of the platform and the wheel by $m_{0}$ and $m_{1}$, respectively; let $\rho_{0}$ and $\rho_{1}$ be the radii of inertia of the platform and the wheel, respectively, relative to the vertical axis passing through their centers of mass; denote the radius of inertia of the wheel relative to its axis of rotation by $\rho_{3}$. Let us introduce the following mass-inertia parameters.

$$
\begin{aligned}
& m=m_{0}+3 m_{1}\left(1+\frac{\rho_{3}^{2}}{2 r^{2}}\right), \\
& m_{s}=m_{0}+3 m_{1}, \quad m_{2}=m-m_{s}=\frac{3 m_{1} \rho_{3}^{2}}{2 r^{2}}, \\
& I_{s}=m_{0}\left(d^{2}+\rho_{0}^{2}\right)+3 m_{1}\left(\rho_{1}^{2}+a^{2}\left(1+\frac{2 \rho_{3}^{2}}{r^{2}}\right)\right) .
\end{aligned}
$$

Three equations of nonholonomic constraints can be obtained which uniquely determine the projections $\dot{x}, \dot{y}$ of the velocity vector of the platform center and its angular velocity $\dot{\psi}$ through the angular velocities of the wheels $\dot{\varphi}_{1}, \dot{\varphi}_{2}$ and $\dot{\varphi}_{3}$ as follows [19]:

$$
\begin{aligned}
& \dot{x}=\frac{2 r}{3} \sum_{i=1}^{3} \sin \left(\psi+(i-1) \frac{2 \pi}{3}\right) \dot{\varphi}_{i}, \\
& \dot{y}=-\frac{2 r}{3} \sum_{i=1}^{3} \cos \left(\psi+(i-1) \frac{2 \pi}{3}\right) \dot{\varphi}_{i}, \\
& \dot{\psi}=-\frac{r}{3 a} \sum_{i=1}^{3} \dot{\varphi}_{i} .
\end{aligned}
$$

The velocity vector of the platform center $C$ is such as $V=\left(V_{x}, V_{y}, \Omega\right)^{T}$, where $V_{x}$ and $V_{y}$ are the components of the $V$ in the body frame $C x_{1} y_{1} z_{1}$; and $\Omega$ is the platform angular velocity.

The kinematic equations which express the relationship between the generalized velocities and pseudo-coordinates of the robot are given by [19]:

$$
\begin{aligned}
\dot{x} & =V_{x} \cos \psi-V_{y} \sin \psi, \\
\dot{y} & =V_{x} \sin \psi+V_{y} \cos \psi, \\
\dot{\psi} & =\Omega, \\
\dot{\varphi}_{1} & =\frac{1}{r}\left(V_{y}+a \Omega\right), \quad \dot{\varphi}_{2}=\frac{1}{2 r}\left(\sqrt{3} V_{x}+V_{y}-2 a \Omega\right), \\
\dot{\varphi}_{3} & =\frac{1}{2 r}\left(-\sqrt{3} V_{x}+V_{y}-2 a \Omega\right) .
\end{aligned}
$$

Using (2.2), we obtain

$$
\begin{aligned}
V_{x} & =\dot{x} \cos \psi+\dot{y} \sin \psi, \\
V_{y} & =-\dot{x} \sin \psi+\dot{y} \cos \psi, \\
\Omega & =\dot{\psi} .
\end{aligned}
$$


The robot dynamic equations can be expressed in the variables $V_{x}, V_{y}$ and $\Omega$ as follows [19]:

$$
\begin{aligned}
& m \dot{V}_{x}-m_{0} d \sin \alpha \dot{\Omega}-m_{s} \Omega V_{y}-m_{0} d \cos \alpha \Omega^{2}=\frac{\sqrt{3}}{2 r}\left(M_{2}-M_{3}\right), \\
& m \dot{V}_{y}+m_{0} d \cos \alpha \dot{\Omega}+m_{s} \Omega V_{x}-m_{0} d \sin \alpha \Omega^{2}=-\frac{1}{2 r}\left(2 M_{1}-M_{2}-M_{3}\right), \\
& m_{0} d\left(-\sin \alpha \dot{V}_{x}+\cos \alpha \dot{V}_{y}\right)+I_{s} \dot{\Omega}+m_{0} d\left(\cos \alpha V_{x}+\sin \alpha V_{y}\right) \Omega=-\frac{a}{r}\left(M_{1}+M_{2}+M_{3}\right)
\end{aligned}
$$

where $M_{1}, M_{2}$ and $M_{3}$ are input torques applied to the wheels.

Similarly to [19], we assume that the torques produced by each of the three geared motors are linear functions of both the applied voltage and angular rotational velocity of the rotor, i.e.,

$$
M_{j}=c_{u} u_{j}-c_{\nu} \dot{\varphi}_{j}, \quad j=1,2,3,
$$

where $c_{u}$ and $c_{\nu}$ are some constants, $u_{j}$ is the voltage applied to the motor, and $c_{\nu} \dot{\varphi}_{j}$ is the counter electromotive torque, $j=1,2,3$.

From (2.1)-(2.4) one can obtain the following dynamic equations of the robot in the world coordinate frame: $O X Y Z$ :

$$
A\left(q_{3}\right) \ddot{q}+B\left(q_{3}, \dot{q}_{3}\right) \dot{q}=P\left(q_{3}\right) U
$$

where

$$
\begin{gathered}
q=\left(q_{1}, q_{2}, q_{3}\right)^{T}, \quad q_{1}=x, \quad q_{2}=y, \quad q_{3}=\psi \\
U=\left(U_{1}, U_{2}, U_{3}\right)^{T}, \quad U_{i}=\left(c_{u} / r\right) u_{i}, i=1,2,3 \\
A\left(q_{3}\right)=\left(\begin{array}{ccc}
m & 0 & -s\left(q_{3}\right) \\
0 & m & c\left(q_{3}\right) \\
-s\left(q_{3}\right) & c\left(q_{3}\right) & I_{s}
\end{array}\right) \\
B\left(q_{3}, \dot{q}_{3}\right)=\left(\begin{array}{ccc}
h & m_{2} \dot{q}_{3} & -c\left(q_{3}\right) \dot{q}_{3} \\
-m_{2} \dot{q}_{3} & h & -s\left(q_{3}\right) \dot{q}_{3} \\
0 & 0 & 2 a^{2} h
\end{array}\right) \\
P\left(q_{3}\right)=\left(\begin{array}{ccc}
\sin q_{3} & \sin \left(q_{3}+\frac{2 \pi}{3}\right) & \sin \left(q_{3}+\frac{4 \pi}{3}\right) \\
-\cos q_{3} & -\cos \left(q_{3}+\frac{2 \pi}{3}\right) & -\cos \left(q_{3}+\frac{4 \pi}{3}\right) \\
-a & -a
\end{array}\right)
\end{gathered}
$$

$h=3 c_{\nu} /\left(2 r^{2}\right), c\left(q_{3}\right)=m_{0} d \cos \left(\alpha+q_{3}\right), s\left(q_{3}\right)=m_{0} d \sin \left(\alpha+q_{3}\right)$. Note that parameter $h$ expresses the robot damping forces.

Let $q=q^{(0)}(t)$ be a reference trajectory which is a twice continuously differentiable function such that the following inequalities hold:

$$
\begin{array}{ll}
\left|q_{k}^{(0)}(t)\right| \leqslant \xi_{1}, & \left|\dot{q}_{k}^{(0)}(t)\right| \leqslant \eta_{1}, \quad\left|\ddot{q}_{k}^{(0)}(t)\right| \leqslant \zeta_{1} \quad(k=1,2), \\
\left|\dot{q}_{3}^{(0)}(t)\right| \leqslant \eta_{2}, \quad\left|\ddot{q}_{3}^{(0)}(t)\right| \leqslant \zeta_{2},
\end{array}
$$

where $\xi_{1}, \eta_{k}$ and $\zeta_{k}$ are some positive reals, $k=1,2$.

The trajectory tracking control problem to be addressed is to construct the controller $U=U(t, q, \dot{q})$ in such a way that the reference trajectory $q^{(0)}(t)$ of the robot (2.6) is globally uniformly asymptotically stable. 


\section{The Problem Solution}

\subsection{The unbounded controller design}

We choose the controller as follows:

$$
U=P^{-1}\left(q_{3}\right)\left(U^{(0)}+U^{(1)}+U^{(2)}\right)
$$

where $P^{-1} P=E$

$$
\begin{aligned}
P^{-1}\left(q_{3}\right)=\frac{2}{3}\left(\begin{array}{ccc}
\sin q_{3} & -\cos q_{3} & -\frac{1}{2 a} \\
\sin \left(q_{3}+\frac{2 \pi}{3}\right)-\cos \left(q_{3}+\frac{2 \pi}{3}\right) & -\frac{1}{2 a} \\
\sin \left(q_{3}+\frac{4 \pi}{3}\right)-\cos \left(q_{3}+\frac{4 \pi}{3}\right) & -\frac{1}{2 a}
\end{array}\right), \\
U^{(0)}=A\left(q_{3}\right) \ddot{q}^{(0)}(t)+B\left(q_{3}, \dot{q}_{3}^{(0)}(t)\right) \dot{q}^{(0)}(t), \\
U^{(1)}=\left(U_{1}^{(1)}, U_{2}^{(1)}, U_{3}^{(1)}\right)^{T}, \\
U_{j}^{(1)}=-\mu_{j}\left(\dot{q}_{j}-\dot{q}_{1}^{(0)}(t)\right), j=1,2,3, \\
U^{(2)}=\left(U_{1}^{(2)}, U_{2}^{(2)}, U_{3}^{(2)}\right)^{T}, \\
U_{j}^{(2)}=-\gamma_{j}\left(q_{j}-q_{j}^{(0)}(t)\right), j=1,2,3,
\end{aligned}
$$

where the constants $\gamma_{j}, j=1,2,3$ are some positive reals and $\mu_{j}, j=1,2,3$ satisfy the following inequalities:

$$
\begin{aligned}
& h+\mu_{j} \geqslant \varepsilon, \quad j=1,2, \\
& 4\left(2 a^{2}(h-\varepsilon)+\mu_{3}\right) \geqslant\left(m_{0} d \eta_{2}+m_{2} \eta_{1}\right)^{2}\left(\frac{1}{h-\varepsilon+\mu_{1}}+\frac{1}{h-\varepsilon+\mu_{2}}\right) .
\end{aligned}
$$

Here $\varepsilon$ is some positive real.

Let us define the state trajectory tracking errors as follows:

$$
\begin{array}{ll}
e_{q}=q-q^{(0)}(t), & \dot{e}_{q}=\dot{q}-\dot{q}^{(0)}(t), \\
e_{q}=\left(e_{1}, e_{2}, e_{3}\right)^{T}, & \dot{e}_{q}=\left(\dot{e}_{1}, \dot{e}_{2}, \dot{e}_{3}\right)^{T} .
\end{array}
$$

The error dynamics equations are given by

$$
A\left(q_{3}^{(0)}(t)+e_{3}\right) \ddot{e}_{q}+B\left(q_{3}^{(0)}(t)+e_{3}, \dot{q}_{3}^{(0)}(t)+\dot{e}_{3}\right) \dot{e}_{q}+f\left(t, e_{3}\right) \dot{e}_{3}=-\mu \dot{e}_{q}-\gamma e_{q},
$$

where $f=\left(m_{2} \dot{q}_{2}^{(0)}(t)-c\left(q_{3}^{(0)}(t)+e_{3}\right) \dot{q}_{3}^{(0)}(t),-s\left(q_{3}^{(0)}(t)+e_{3}\right) \dot{q}_{3}^{(0)}(t)-m_{2} \dot{q}_{1}^{(0)}(t), 0\right)^{T}$, $\mu=\operatorname{diag}\left(\mu_{1}, \mu_{2}, \mu_{3}\right), \gamma(x)=\operatorname{diag}\left(\gamma_{1}, \gamma_{2}, \gamma_{3}\right)$.

Note that the system of equations (3.5) is precompact [27] and the corresponding system of limiting equations has a similar form

$$
A\left(q_{3}^{(0) *}(t)+e_{3}\right) \ddot{e}_{q}+B\left(q_{3}^{(0) *}(t)+e_{3}, \dot{q}_{3}^{(0) *}(t)+\dot{e}_{3}\right) \dot{e}_{q}+f^{*}\left(t, e_{3}\right) \dot{e}_{3}=-\mu \dot{e}_{q}-\gamma e_{q},
$$

where $q_{3}^{(0) *}(t)=\lim _{n \rightarrow \infty} q_{3}^{(0)}\left(t_{n}+t\right), \dot{q}_{3}^{(0) *}(t)=\lim _{n \rightarrow \infty} \dot{q}_{3}^{(0)}\left(t_{n}+t\right)$ and $f^{*}\left(t, e_{3}\right)=\lim _{n \rightarrow \infty} f\left(t_{n}+t, e_{3}\right)$. 
Theorem 1. Consider the closed-loop system (3.5). Let inequalities (3.3) hold. Then the origin $\dot{x}=x=0$ of (3.5) is globally uniformly asymptotically stable.

Proof. As a Lyapunov function candidate $V=V\left(t, e_{q}, \dot{e}_{q}\right)$, we consider the following function:

$$
V=\frac{1}{2} \dot{e}_{q}^{T} A\left(q_{3}^{(0)}(t)+e_{3}\right) \dot{e}_{q}+\frac{1}{2}\left(\gamma_{1} e_{1}^{2}+\gamma_{2} e_{2}^{2}+\gamma_{3} e_{3}^{2}\right)
$$

One can easily see that $V$ is positive definite, radially unbounded and the following holds:

$$
V\left(t, e_{q}, \dot{e}_{q}\right) \rightarrow 0 \text { uniformly on } t \in \mathbb{R} \text { as }\left(e_{q}, \dot{e}_{q}\right) \rightarrow 0 \text {. }
$$

The time derivative of the function (3.7) is given by

$$
\dot{V}=\frac{1}{2} \dot{e}_{q}^{T} \dot{A}\left(q_{3}^{(0)}(t)+e_{3}\right) \dot{e}_{q}+\dot{e}_{q}^{T} A\left(q_{3}^{(0)}(t)+e_{3}\right) \ddot{e}_{q}+\gamma_{1} e_{1} \dot{e}_{1}+\gamma_{2} e_{2} \dot{e}_{2}+\gamma_{3} e_{3} \dot{e}_{3}
$$

From (3.8), using (3.5), one can see that the time derivative of the function (3.7) becomes

$$
\begin{aligned}
\dot{V} & =\frac{1}{2}\left(\dot{q}_{3}^{(0)}(t)+\dot{e}_{3}\right)\left(\dot{e}_{1}, \dot{e}_{2}, \dot{e}_{3}\right)\left(\begin{array}{ccc}
0 & 0 & -c\left(q_{3}\right) \\
0 & 0 & -s\left(q_{3}\right) \\
-c\left(q_{3}\right) & -s\left(q_{3}\right) & 0
\end{array}\right)\left(\begin{array}{c}
\dot{e}_{1} \\
\dot{e}_{2} \\
\dot{e}_{3}
\end{array}\right)- \\
& -\dot{e}_{3}\left(\dot{e}_{1}, \dot{e}_{2}, \dot{e}_{3}\right)\left(\begin{array}{ccc}
0 & m_{2} & -c\left(q_{3}\right) \\
-m_{2} & 0 & -s\left(q_{3}\right) \\
0 & 0 & 0
\end{array}\right)\left(\begin{array}{c}
\dot{q}_{1}^{(0)}(t) \\
\dot{q}_{2}^{(0)}(t) \\
\dot{q}_{3}^{(0)}(t)
\end{array}\right)- \\
& -\left(\dot{e}_{3}+\dot{q}_{3}^{(0)}(t)\right)\left(\dot{e}_{1}, \dot{e}_{2}, \dot{e}_{3}\right)\left(\begin{array}{ccc}
0 & m_{2} & -c\left(q_{3}\right) \\
-m_{2} & 0 & -s\left(q_{3}\right) \\
0 & 0 & 0
\end{array}\right)\left(\begin{array}{c}
\dot{e}_{1} \\
\dot{e}_{2} \\
\dot{e}_{3}
\end{array}\right)- \\
& -\left(\dot{e}_{1}, \dot{e}_{2}, \dot{e}_{3}\right) \operatorname{diag}\left(h+\mu_{1}, h+\mu_{2}, 2 a^{2} h+\mu_{3}\right)\left(\begin{array}{c}
\dot{e}_{1} \\
\dot{e}_{2} \\
\dot{e}_{3}
\end{array}\right)
\end{aligned}
$$

One can easily see that the following equality holds:

$$
\begin{gathered}
\frac{1}{2}\left(\dot{q}_{3}^{(0)}(t)+\dot{e}_{3}\right)\left(\dot{e}_{1}, \dot{e}_{2}, \dot{e}_{3}\right)\left(\begin{array}{ccc}
0 & 0 & -c\left(q_{3}\right) \\
0 & 0 & -s\left(q_{3}\right) \\
-c\left(q_{3}\right) & -s\left(q_{3}\right) & 0
\end{array}\right)\left(\begin{array}{c}
\dot{e}_{1} \\
\dot{e}_{2} \\
\dot{e}_{3}
\end{array}\right)- \\
-\left(\dot{e}_{3}+\dot{q}_{3}^{(0)}(t)\right)\left(\dot{e}_{1}, \dot{e}_{2}, \dot{e}_{3}\right)\left(\begin{array}{ccc}
0 & m_{2} & -c\left(q_{3}\right) \\
-m_{2} & 0 & -s\left(q_{3}\right) \\
0 & 0 & 0
\end{array}\right)\left(\begin{array}{c}
\dot{e}_{1} \\
\dot{e}_{2} \\
\dot{e}_{3}
\end{array}\right) \equiv 0 .
\end{gathered}
$$


From (3.9), using (3.10), one can obtain

$$
\begin{aligned}
\dot{V} & =-\dot{e}_{3}\left(\dot{e}_{1}, \dot{e}_{2}, \dot{e}_{3}\right)\left(\begin{array}{ccc}
0 & m_{2} & -c\left(q_{3}\right) \\
-m_{2} & 0 & -s\left(q_{3}\right) \\
0 & 0 & 0
\end{array}\right)\left(\begin{array}{c}
\dot{q}_{1}^{(0)}(t) \\
\dot{q}_{2}^{(0)}(t) \\
\dot{q}_{3}^{(0)}(t)
\end{array}\right)- \\
& -\left(\dot{e}_{1}, \dot{e}_{2}, \dot{e}_{3}\right) \operatorname{diag}\left(h+\mu_{1}, h+\mu_{2}, 2 a^{2} h+\mu_{3}\right)\left(\begin{array}{c}
\dot{e}_{1} \\
\dot{e}_{2} \\
\dot{e}_{3}
\end{array}\right) .
\end{aligned}
$$

Using (3.11), one can easily see that the time-derivative of $V$ can be written as

$$
\begin{aligned}
\dot{V} & =-\left(h+\mu_{1}\right) \dot{e}_{1}^{2}-\left(h+\mu_{2}\right) \dot{e}_{2}^{2}-\left(2 a^{2} h+\mu_{3}\right) \dot{e}_{3}^{2}+ \\
& +\left(m_{0} d \dot{q}_{3}^{(0)}(t) \cos \left(\alpha+e_{3}+q_{3}^{(0)}(t)\right)-m_{2} \dot{q}_{2}^{(0)}(t)\right) \dot{e}_{1} \dot{e}_{3}+ \\
& +\left(m_{2} \dot{q}_{1}^{(0)}(t)+m_{0} d \dot{q}_{3}^{(0)}(t) \sin \left(\alpha+e_{3}+q_{3}^{(0)}(t)\right)\right) \dot{e}_{2} \dot{e}_{3} .
\end{aligned}
$$

From (3.12), using (2.7), one can obtain the following estimation of $\dot{V}$ :

$$
\dot{V} \leqslant-\left(h+\mu_{1}\right) \dot{e}_{1}^{2}-\left(h+\mu_{2}\right) \dot{e}_{2}^{2}-\left(2 a^{2} h+\mu_{3}\right) \dot{e}_{3}^{2}+\left(m_{0} d \eta_{2}+m_{2} \eta_{1}\right)\left|\dot{e}_{3}\right|\left(\left|\dot{e}_{1}\right|+\left|\dot{e}_{2}\right|\right) \text {. }
$$

From (3.13), using (3.3), one can obtain

$$
\dot{V} \leqslant-\varepsilon\left(\dot{e}_{1}^{2}+\dot{e}_{2}^{2}+2 a^{2} \dot{e}_{3}^{2}\right) \leqslant 0 .
$$

Indeed, rewrite the estimation (3.13) as

$$
\begin{aligned}
\dot{V} & \leqslant-\left(h+\mu_{1}-\varepsilon\right) \dot{e}_{1}^{2}-\left(h+\mu_{2}-\varepsilon\right) \dot{e}_{2}^{2}-\left(2 a^{2}(h-\varepsilon)+\mu_{3}\right) \dot{e}_{3}^{2}+ \\
& +\left(m_{0} d \eta_{2}+m_{2} \eta_{1}\right)\left|\dot{e}_{3}\right|\left(\left|\dot{e}_{1}\right|+\left|\dot{e}_{2}\right|\right)-\varepsilon\left(\dot{e}_{1}^{2}+\dot{e}_{2}^{2}+2 a^{2} \dot{e}_{3}^{2}\right) .
\end{aligned}
$$

Note that the quadratic form

$-\left(h+\mu_{1}-\varepsilon\right) \dot{e}_{1}^{2}-\left(h+\mu_{2}-\varepsilon\right) \dot{e}_{2}^{2}-\left(2 a^{2}(h-\varepsilon)+\mu_{3}\right) \dot{e}_{3}^{2}+\left(m_{0} d \eta_{2}+m_{2} \eta_{1}\right)\left|\dot{e}_{3}\right|\left(\left|\dot{e}_{1}\right|+\left|\dot{e}_{2}\right|\right)$

is negative semidefinite if inequalities (3.3) hold.

Then one can obtain the estimation (3.14). From (3.14) one can easily see that $\dot{V}$ is a negative semidefinite function. Using the Persidskii theorem on uniform stability [25], one can easily find that the zero solution $\dot{e}_{q}=e_{q}=0$ of the system (3.5) is uniformly stable.

To obtain the uniform global attractivity property of the zero solution $\dot{e}_{q}=e_{q}=0$ of the system (3.5), we note that the set $\left\{\left(e_{q}, \dot{e}_{q}\right) \in \mathbb{R}^{6}: \dot{e}_{1}=\dot{e}_{2}=\dot{e}_{3}=0\right\}$ does not contain the solutions of the system (3.6) except for $e_{1}=e_{2}=e_{3}=0$.

Then, using the theorem on asymptotic stability from [2], we find that the zero solution $\dot{e}_{q}=e_{q}=0$ of the system (3.5) is globally uniformly asymptotically stable. In other words, the controller (3.1), (3.2) solves the global trajectory tracking problem of the robot (2.6). This completes the proof.

REMARK 1. Note that the structure of the controller (3.1), (3.2) is similar to that proposed in [21] for robotic manipulators. This control structure was used in [24] for a simpler model of an omnidirectional mobile robot where the mass center of the platform coincides with its geometrical center. 


\subsection{The bounded controller design}

Consider the following controller:

$$
U=P^{-1}\left(q_{3}\right)\left(U^{(0)}+U^{(2)}\right),
$$

where the term $U^{(2)}$ is bounded and defined as

$$
U_{j}^{(2)}=-\gamma_{j} \arctan \left(q_{j}-q_{j}^{(0)}(t)\right), j=1,2,3,
$$

where $\gamma_{j}>0, j=1,2,3$.

The error dynamics equations are

$$
\begin{gathered}
A\left(q_{3}^{(0)}(t)+e_{3}\right) \ddot{e}_{q}+B\left(q_{3}^{(0)}(t)+e_{3}, \dot{q}_{3}^{(0)}(t)+\dot{e}_{3}\right) \dot{e}_{q}+f\left(t, e_{3}\right) \dot{e}_{3}= \\
=-\operatorname{diag}\left(\gamma_{1} \arctan e_{1}, \gamma_{2} \arctan e_{2}, \gamma_{3} \arctan e_{3}\right) .
\end{gathered}
$$

Theorem 2. Consider the closed-loop system (3.18). Let the damping force of the robot be large enough that the following inequality holds:

$$
h \geqslant \varepsilon+\frac{m_{0} d \eta_{2}+m_{2} \eta_{1}}{2 a}
$$

where $\varepsilon$ is some positive real.

Then the origin $\dot{e}_{q}=e_{q}=0$ of (3.18) is globally uniformly asymptotically stable.

Proof. As a Lyapunov function candidate, we choose the following function:

$$
V=\frac{1}{2} \dot{e}_{q}^{T} A\left(q_{3}^{(0)}(t)+e_{3}\right) \dot{e}_{q}+\sum_{i=1}^{3} \gamma_{i} \int_{0}^{e_{i}} \arctan e_{i} d e_{i} .
$$

One can easily see that $V$ is positive definite, radially unbounded and the following holds:

$$
V\left(t, e_{q}, \dot{e}_{q}\right) \rightarrow 0 \text { uniformly on } t \in \mathbb{R} \text { as }\left(e_{q}, \dot{e}_{q}\right) \rightarrow 0 .
$$

The time derivative of the function (3.20) is given by

$$
\dot{V}=\frac{1}{2} \dot{e}_{q}^{T} \dot{A}\left(q_{3}^{(0)}(t)+e_{3}\right) \dot{e}_{q}+\dot{e}_{q}^{T} A\left(q_{3}^{(0)}(t)+e_{3}\right) \ddot{e}_{q}+\sum_{i=1}^{3} \gamma_{i} \dot{e}_{i} \arctan e_{i} .
$$

One can easily see that the time derivative (3.21) satisfies (3.12), where $\mu_{1}=\mu_{2}=\mu_{3}=0$. Then one can obtain

$$
\dot{V} \leqslant-h\left(\dot{e}_{1}^{2}+\dot{e}_{2}^{2}\right)-2 a^{2} h \dot{e}_{3}^{2}+\left(m_{0} d \eta_{2}+m_{2} \eta_{1}\right)\left|\dot{e}_{3}\right|\left(\left|\dot{e}_{1}\right|+\left|\dot{e}_{2}\right|\right) .
$$

From (3.22), using (3.19), one can obtain the following estimation:

$$
\dot{V} \leqslant-\varepsilon\left(\dot{e}_{1}^{2}+\dot{e}_{2}^{2}+2 a^{2} \dot{e}_{3}^{2}\right) \leqslant 0 .
$$

To prove the estimation (3.23), rewrite (3.13) as follows:

$$
\begin{aligned}
\dot{V} & \leqslant-(h-\varepsilon)\left(\dot{e}_{1}^{2}+\dot{e}_{2}^{2}\right)-2 a^{2}(h-\varepsilon) \dot{e}_{3}^{2}+ \\
& +\left(m_{0} d \eta_{2}+m_{2} \eta_{1}\right)\left|\dot{e}_{3}\right|\left(\left|\dot{e}_{1}\right|+\left|\dot{e}_{2}\right|\right)-\varepsilon\left(\dot{e}_{1}^{2}+\dot{e}_{2}^{2}+2 a^{2} \dot{e}_{3}^{2}\right) .
\end{aligned}
$$

Note that the quadratic form

$$
-(h-\varepsilon)\left(\dot{e}_{1}^{2}+\dot{e}_{2}^{2}+2 a^{2} \dot{e}_{3}^{2}\right)+\left(m_{0} d \eta_{2}+m_{2} \eta_{1}\right)\left|\dot{e}_{3}\right|\left(\left|\dot{e}_{1}\right|+\left|\dot{e}_{2}\right|\right)
$$

is negative semidefinite if inequality (3.19) holds.

From (3.23), using the theorem on asymptotic stability from [2], we get the proof. 


\section{Numerical results}

In this section, the performance of the proposed controllers is illustrated.

The robot parameters are given as

$$
\begin{aligned}
& m_{0}=20 \mathrm{~kg}, \quad m_{1}=1 \mathrm{~kg}, \\
& r=0.1 \mathrm{~m}, \quad a=0.25 \mathrm{~m}, \quad d=0.05 \mathrm{~m}, \\
& \alpha=\pi / 6 \mathrm{rad}, \quad c_{\nu}=6 \cdot 10^{-5} \mathrm{~N} \cdot \mathrm{m} \cdot \mathrm{sec}, \\
& \rho_{0}=0.17 \mathrm{~m}, \quad \rho_{1}=0.05 \mathrm{~m}, \quad \rho_{3}=0.07 \mathrm{~m} .
\end{aligned}
$$

Consider the ellipse tracking of the robot with a constant relative angular velocity of the platform. Choose the desired trajectory as follows:

$$
q_{1}^{(0)}(t)=1+20 \cos t \mathrm{~m}, \quad q_{2}^{(0)}(t)=2+30 \sin t \mathrm{~m}, \quad q_{3}^{(0)}(t)=\pi / 4+10 t \mathrm{rad} .
$$

The control gain matrices $\operatorname{diag}\left(\gamma_{1}, \gamma_{2}, \gamma_{3}\right)$ and $\operatorname{diag}\left(\mu_{1}, \mu_{2}, \mu_{3}\right)$ of the controller (3.1), (3.2) were chosen such that

$$
\begin{aligned}
& \gamma_{1}=25 \mathrm{~N} / \mathrm{m}, \quad \gamma_{2}=25 \mathrm{~N} / \mathrm{m}, \quad \gamma_{3}=20 \mathrm{~N} \cdot \mathrm{m}, \\
& \mu_{1}=35 \mathrm{~N} \cdot \mathrm{sec} / \mathrm{m}, \quad \mu_{2}=35 \mathrm{~N} \cdot \mathrm{sec} / \mathrm{m}, \quad \mu_{3}=20 \mathrm{~N} \cdot \mathrm{m} \cdot \mathrm{sec} .
\end{aligned}
$$

In order to check the property of global tracking, we consider the simulations results using the initial conditions for the robot such that

$$
\begin{array}{llll}
q_{1}(0) & =51 \mathrm{~m}, & q_{2}(0)=-68 \mathrm{~m}, & q_{3}(0)=-2.21 \mathrm{rad}, \\
\dot{q}_{1}(0)=25 \mathrm{~m} / \mathrm{sec}, & \dot{q}_{2}(0)=-15 \mathrm{~m} / \mathrm{sec}, & \dot{q}_{3}(0)=20 \mathrm{rad} / \mathrm{sec} .
\end{array}
$$

The tracking performance of the controller (3.1), (3.2) within the time interval $t=50 \mathrm{sec}$ is shown in Figs. 2 and 3. From these results, it can be seen that the controller (3.1), (3.2) provides the convergence to the reference trajectory of the robot. The time evolution of the control inputs is shown in Fig. 4.

To verify the effectiveness of the bounded controller (3.16)-(3.17), the gain matrix is set as

$$
\operatorname{diag}\left(\gamma_{1}, \gamma_{2}, \gamma_{3}\right)=\operatorname{diag}(32,32,1.5) .
$$

Let $h=9 \mathrm{~N} \cdot \mathrm{sec} / \mathrm{m}$ and $t=120 \mathrm{sec}$.

The simulation results of the controller (3.16)-(3.17) are shown in Figs. 5 and 6 for the following initial conditions:

$$
\begin{array}{llll}
q_{1}(0)=101 \mathrm{~m}, & q_{2}(0)=-98 \mathrm{~m}, & q_{3}(0)=-2.21 \mathrm{rad}, \\
\dot{q}_{1}(0)=25 \mathrm{~m} / \mathrm{sec}, & \dot{q}_{2}(0)=-15 \mathrm{~m} / \mathrm{sec}, & \dot{q}_{3}(0)=20 \mathrm{rad} / \mathrm{sec} .
\end{array}
$$

The control inputs are shown in Fig. 7.

In order to compare the performances, the simulation tests were also performed with the "PD+" like controller proposed in [24] for a simpler robot model:

$$
\begin{aligned}
U & =P^{-1}\left(q_{3}\right)\left(U^{(0)}+U^{(2)}\right), \\
U^{(0)} & =A\left(q_{3}\right) \ddot{q}^{(0)}(t)+B\left(q_{3}, \dot{q}_{3}^{(0)}(t)\right) \dot{q}^{(0)}(t), \\
U_{j}^{(2)} & =-\gamma_{j}\left(q_{j}-q_{j}^{(0)}(t)\right), \quad j=1,2,3 .
\end{aligned}
$$




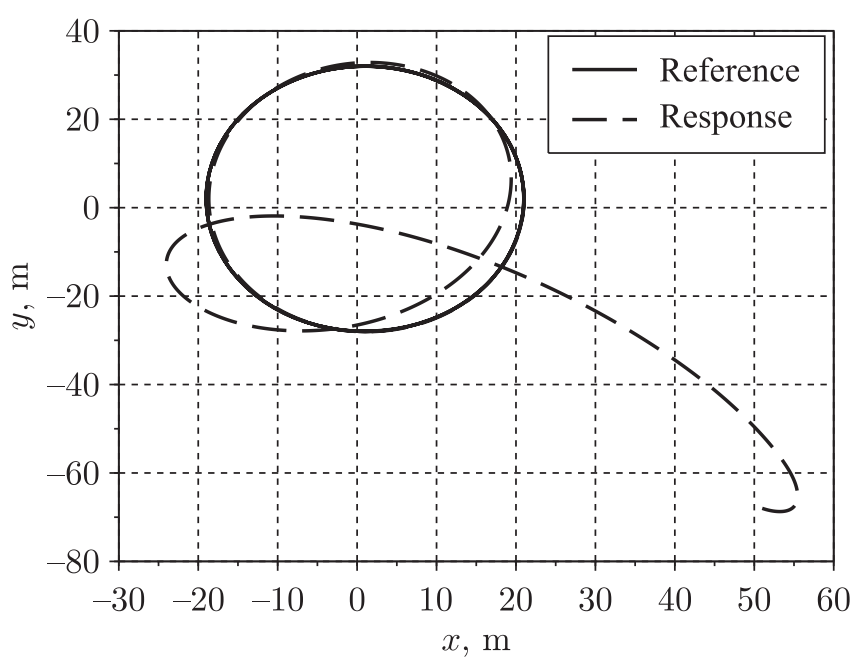

Fig. 2. The robot's trajectory tracking using the controller (3.1)-(3.2).

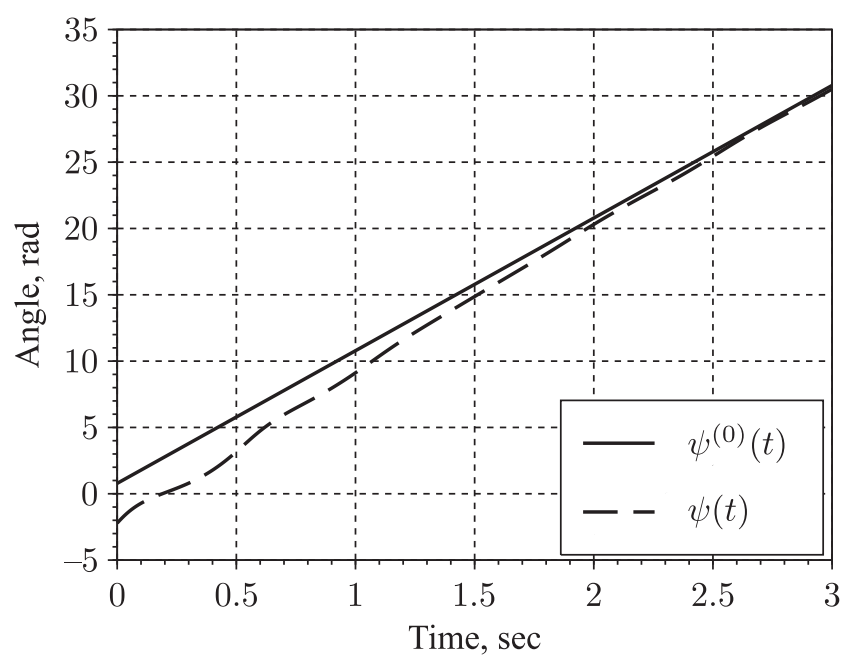

Fig. 3. Evolution of the robot's angular position using the controller (3.1)-(3.2).

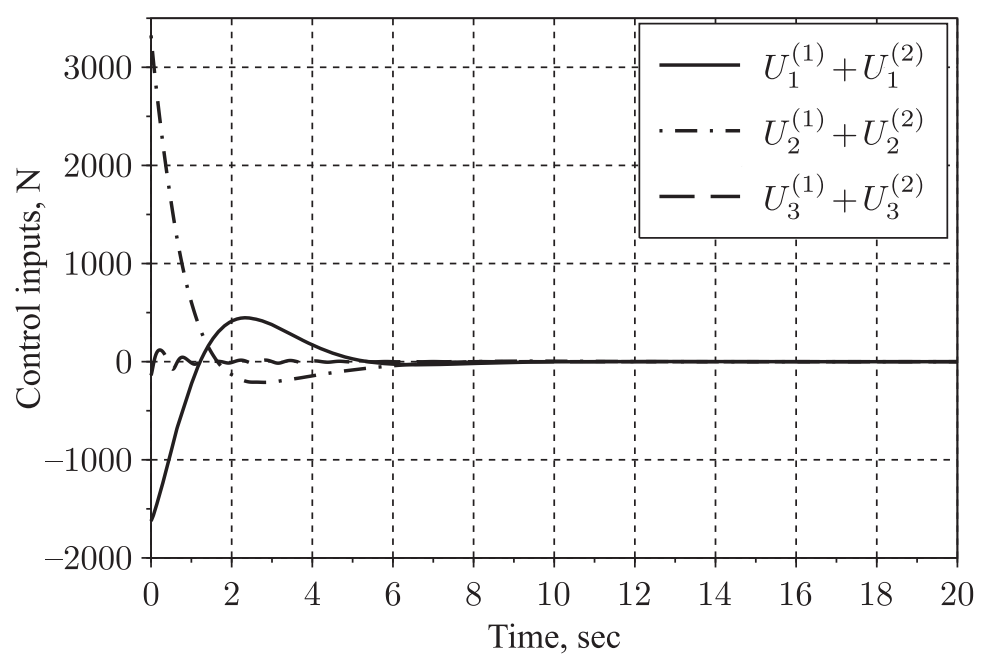

Fig. 4. Stabilizing terms of control inputs (3.1), (3.2). 


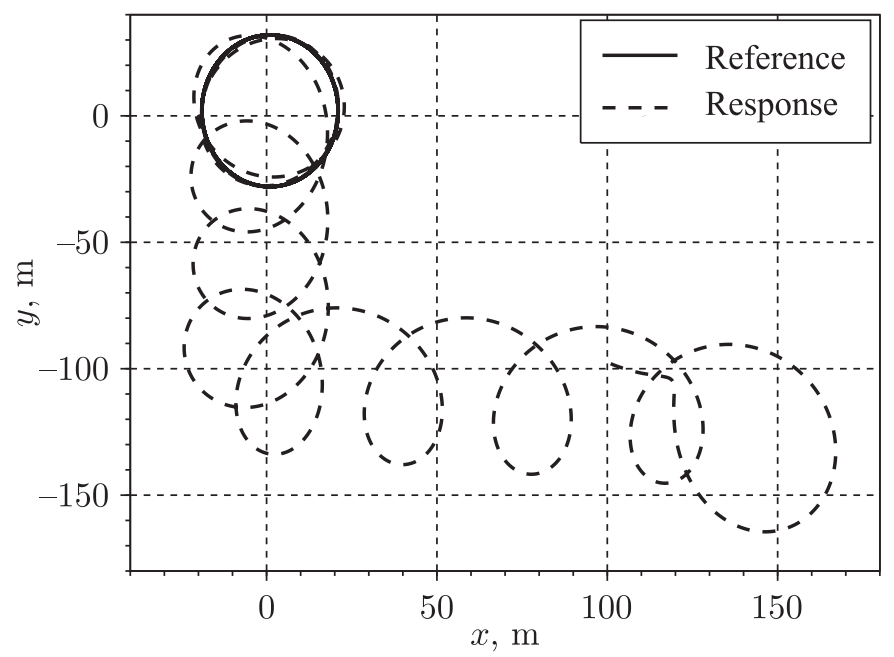

Fig. 5. The robot's trajectory tracking using the controller (3.16)-(3.17).

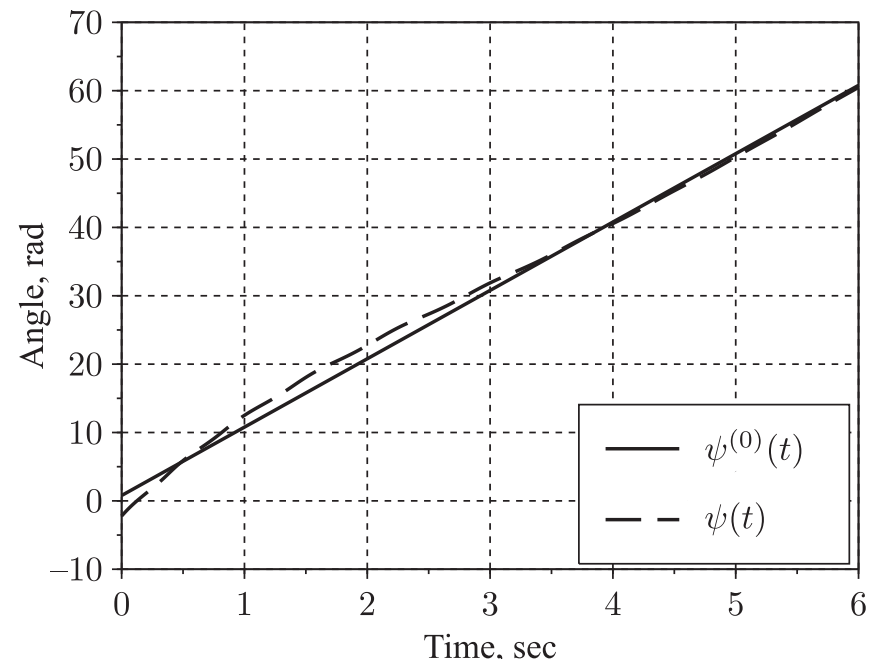

Fig. 6. Evolution of the robot's angular position using the controller (3.16)-(3.17).

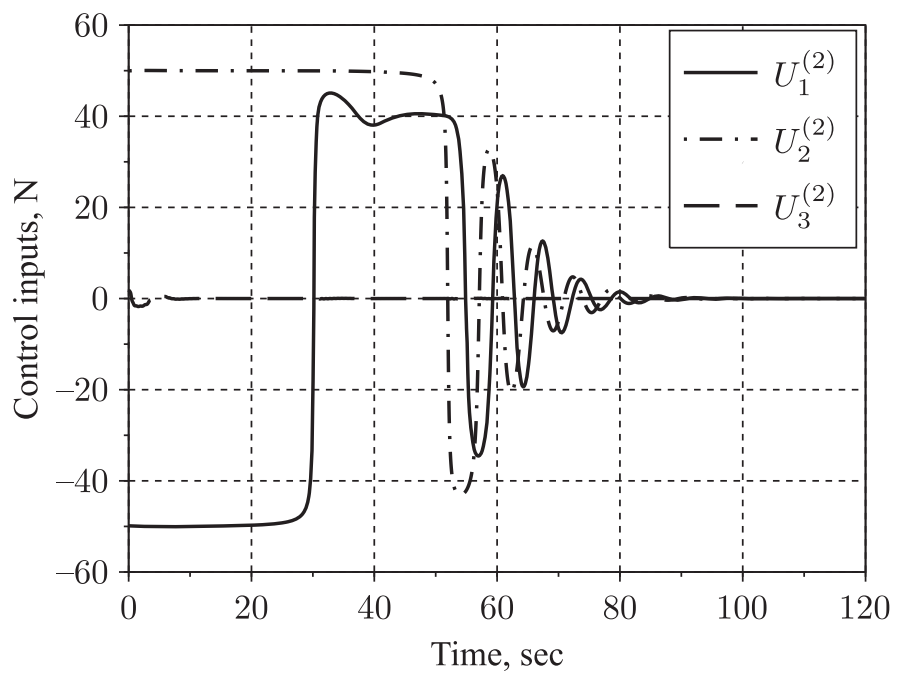

Fig. 7. Stabilizing terms of control inputs (3.16), (3.17). 
We fixed the control gain parameters to $\gamma_{1}=\gamma_{2}=0.5, \gamma_{3}=0.4$ using the trial and error approach.

Figures 8 and 9 show the simulation results for the controller (4.7), using the initial conditions (4.11). The control inputs are shown in Fig. 10. Analyzing the graphs in Figs. 8 and 9, we can see that the controller (4.7) does not ensure the trajectory tracking of the robot during the time interval $t=120 \mathrm{sec}$. In order to track the system (2.6), (4.7) along the reference trajectory (4.2), it is necessary to substantially increase some control gains parameters. Namely, we have to fix the control gain parameters to the following values:

$$
\gamma_{1}=1, \quad \gamma_{2}=1, \quad \gamma_{3}=0.4
$$

From these results, it can be seen that the controller (3.16), (3.17), (4.5) provides the convergence to the reference angular coordinate of the platform.

The control inputs (4.7), (4.8) and the robot's trajectory tracking are shown in Figs. 11 and 12 , respectively.

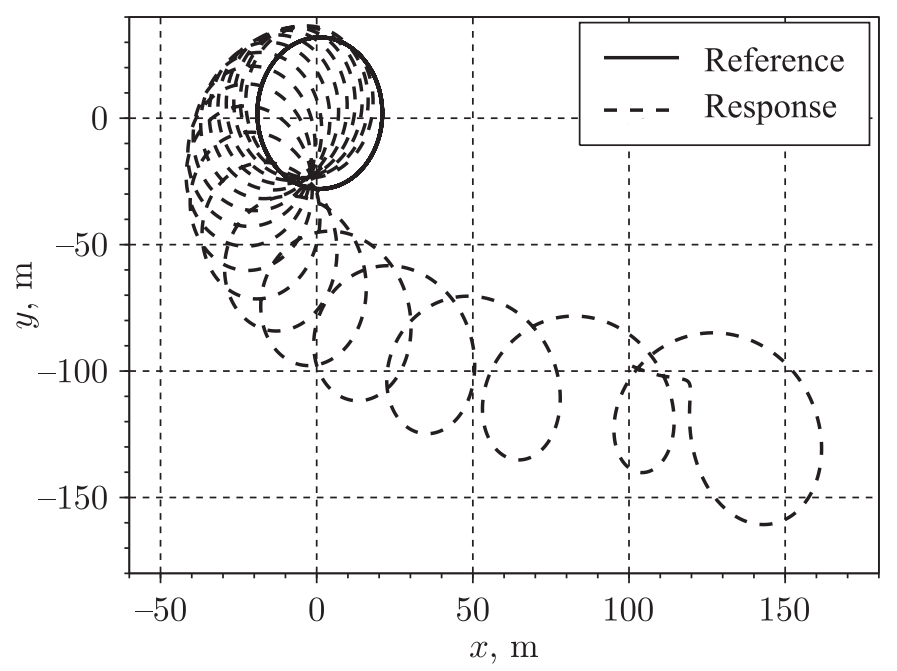

Fig. 8. The robot's trajectory tracking using the controller (4.7).

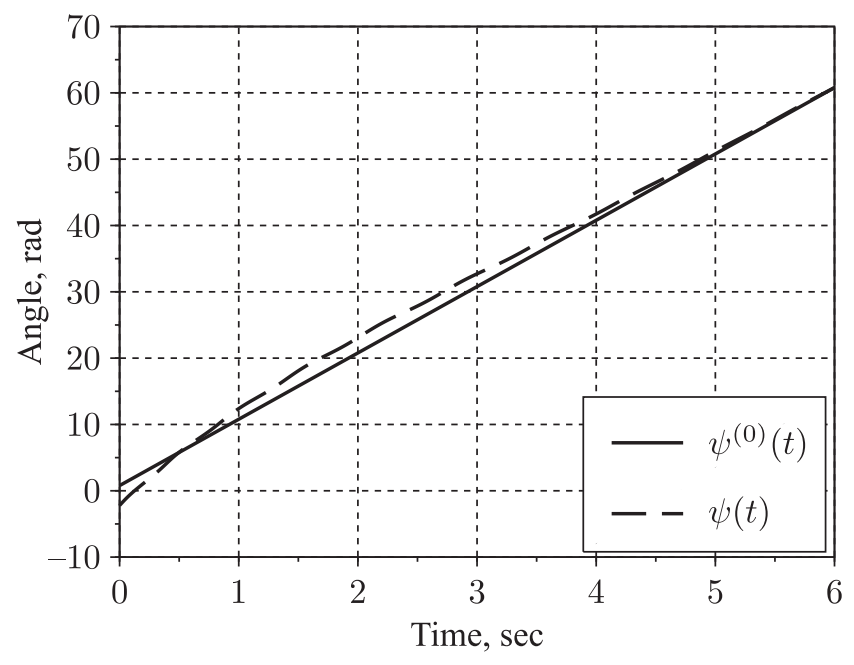

Fig. 9. Evolution of the robot's angular position using the controller (4.7). 


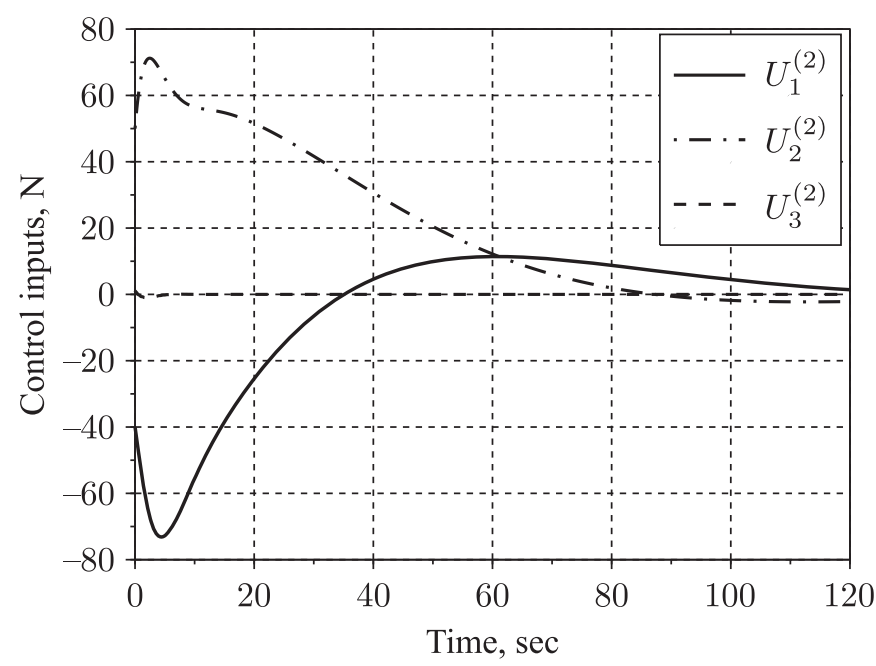

Fig. 10. Stabilizing terms of control inputs (4.7).

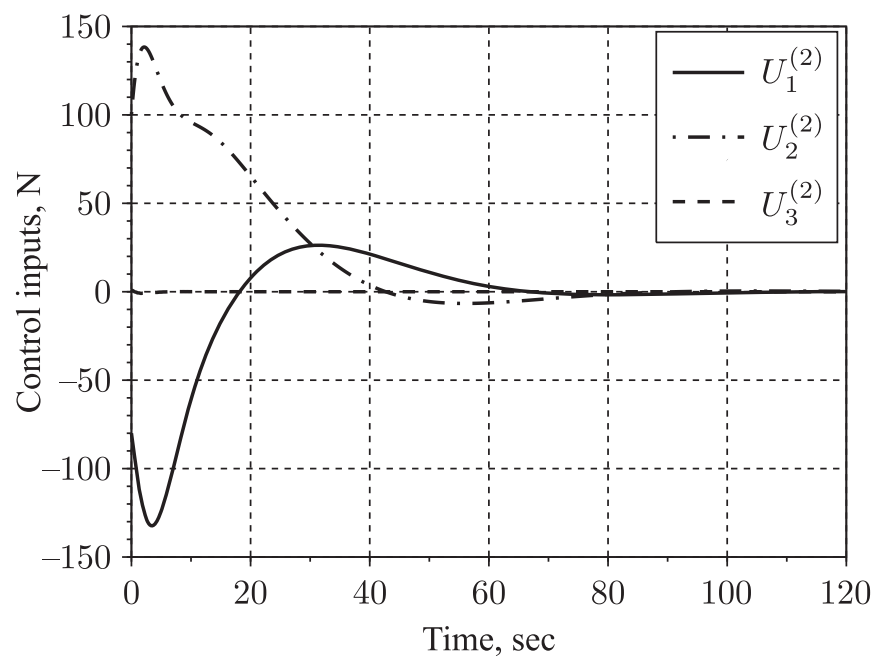

Fig. 11. Stabilizing terms of control inputs (4.7), (4.8).

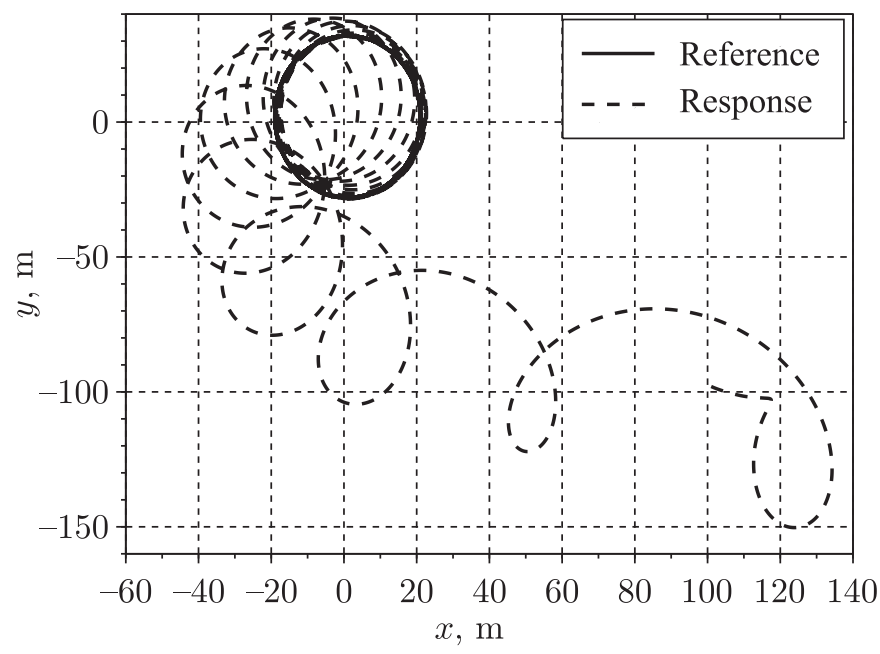

Fig. 12. The robot's trajectory tracking using the controller (4.7)-(4.8). 
Note that our controller (3.16), (3.17), (4.5) requires a significantly less control energy than (4.7), (4.8). To measure the control energy, we used the integral of the squared input (ISI) index given by

$$
I S I=\int_{0}^{T}\|U(t)\|^{2} d t
$$

where $T$ is the simulation time, $U(t)=P^{-1}\left(q_{3}(t)\right)\left(U^{(0)}\left(t, q_{3}(t)\right)+U^{(2)}(t, q(t))\right)$.

Table 1 shows the values of ISI. One can see from Table 1 that the lowest ISI value corresponds to the controller (3.16), (3.17), (4.5). Thus, the comparative analysis has confirmed that the proposed controller (3.16), (3.17), (4.5) provides better performance than that of [24].

Table 1. Values of ISI index of the controllers (3.16), (3.17), and (4.7), (4.8)

\begin{tabular}{|c|c|c|}
\hline Index & Controller (3.16), (3.17), (4.5) & Controller (4.7), (4.8) \\
\hline ISI & 20682479 & 20744258 \\
\hline
\end{tabular}

Compare also the performances of the controller (3.16)-(3.17) and that obtained in [6] and expressed as follows:

$$
U=P^{-1}\left(q_{3}\right)\left(U^{(0)}+U^{(2)}\right)
$$

where the term $U^{(2)}$ is defined as

$$
U_{j}^{(2)}=-\gamma_{j} \arctan \left(q_{j}-q_{j}^{(0)}(t)\right), j=1,2, \quad U_{3}^{(2)}=-\gamma_{3} \sin \frac{q_{3}-q_{3}^{(0)}(t)}{2},
$$

where $\gamma_{j}>0, j=1,2,3$.

For the controller (4.9)-(4.10) we fixed the gain parameters (4.5) which are equal to those of the controller (3.16)-(3.17).

In addition to the ISI index, let us use the root mean square error (RMSE) values of the closed-loop system's position and velocity which are calculated as follows:

$$
\operatorname{RMSE}\left(e_{q}\right)=\sqrt{\frac{1}{T} \int_{0}^{T}\left\|e_{q}(t)\right\|^{2} d t}, \quad \operatorname{RMSE}\left(\dot{e}_{q}\right)=\sqrt{\frac{1}{T} \int_{0}^{T}\left\|\dot{e}_{q}(t)\right\|^{2} d t}
$$

where the state errors $e_{q}$ and $\dot{e}_{q}$ are given by (3.4).

Table 2 shows the values of ISI and RMSE for the initial deviations from the reference trajectory of the robot such that

$$
\begin{array}{lll}
e_{1}(0)=10 \mathrm{~m}, & e_{2}(0)=-10 \mathrm{~m}, & e_{3}(0)=-3 \mathrm{rad}, \\
\dot{e}_{1}(0)=5 \mathrm{~m} / \mathrm{sec}, & \dot{e}_{2}(0)=-5 \mathrm{~m} / \mathrm{sec}, & \dot{e}_{3}(0)=3 \mathrm{rad} / \mathrm{sec} .
\end{array}
$$

Table 2. Values of ISI and RMSE indexes of the controllers (3.16), (3.17) and (4.9), (4.10)

\begin{tabular}{|c|c|c|}
\hline Index & Controller (3.16), (3.17) & Controller (4.9), (4.10) \\
\hline ISI & 8655024.7 & 8705235.5 \\
$\operatorname{RMSE}\left(e_{q}\right)$ & 28.72 & 46.41 \\
$\operatorname{RMSE}\left(\dot{e}_{q}\right)$ & 6.48 & 6.56 \\
\hline
\end{tabular}


As can be seen from Table 2 , the lowest ISI, $\operatorname{RMSE}(x)$ and $\operatorname{RMSE}(\dot{x})$ values correspond to the controller (3.16)-(3.17). Thus, one can conclude that for not very large initial deviations the controller (3.16)-(3.17) is more effective than (4.9), (4.10).

\section{Conclusion}

This paper presents a solution to the global trajectory tracking control problem of an omnidirectional mobile robot with a displaced mass center. The proposed solution relies on the application of two control schemes employing a Lyapunov function with a semidefinite time derivative to the resulting error model. The attractivity property of the zero solution of the closed-loop system has been proved using the quasi-invariance principle for nonautonomous differential equations. The structure of the first proposed controller is a proportional-derivative term plus feedforward. The second controller is a nonlinear bounded proportional term plus feedforward. The simulation tests have shown good performances of the proposed controllers for very large initial errors. It has been shown that the proposed bounded controller is practically more useful than a conventional "PD+" when damping forces of the robot are large enough.

\section{References}

[1] Adamov, B. I., A Study of the Controlled Motion of a Four-Wheeled Mecanum Platform, Russ. J. Nonlinear Dyn., 2018, vol. 14, no. 2, pp. 265-290.

[2] Andreev, A.S., On the Asymptotic Stability and Instability of the Zeroth Solution of a NonAutonomous System, J. Appl. Math. Mech., 1984, vol.48, no. 2, pp.154-160; see also: Prikl. Mat. Mekh., 1984, vol. 48, no. 2, pp. 225-232.

[3] Andreyev, A. S. and Peregudova, O. A., The Motion Control of a Wheeled Mobile Robot, J. Appl. Math. Mech., 2015, vol. 79, no. 4, pp. 316-324.

[4] Andreyev, A. S. and Peregudova, O.A., Trajectory Tracking Problem for Omnidirectional Mobile Robot with Parameter Variations and Delayed Feedback, in Proceedings of the ECCOMAS Thematic Conference on Multibody Dynamics (Barcelona, 2015).

[5] Andreev, A.S. and Peregudova, O.A., Nonlinear Controllers in the Regulation Problem of the Robots, IFAC PapersOnLine, 2018, vol. 51, no. 4, pp. 7-12.

[6] Andreev, A. S. and Peregudova, O. A., On Output Feedback Trajectory Tracking Control of an OmniMobile Robot, IFAC PapersOnLine, 2019, vol. 52, no. 8, pp. 37-42.

[7] Andreev, A. S. and Peregudova, O. A., On Time-Delayed Feedback Trajectory Tracking Control of a Mobile Robot with Omni-Wheels, in Proc. of the 12th International Workshop on Robot, Motion and Control (Poznań University of Technology, Poznań, Poland, 8-10 July, 2019), pp. 143-147.

[8] Araújo, H.X., Conceiçāo, A. G.S., Oliveira, G.H. C., and Pitanga, J., Model Predictive Control Based on LMIs Applied to an Omni-Directional Mobile Robot, in IFAC Proc. Vol., 2011, vol.44, no. 1, pp. 8171-8176.

[9] Ashmore, M. and Barnes, N., Omni-Drive Robot Motion on Curved Paths: The Fastest Path between Two Points Is Not a Straight-Line, in AI 2002: Advances in Artificial Intelligence, Lecture Notes in Comput. Sci., vol. 2557, Berlin: Springer, 2002, pp. 225-236.

[10] Borisov, A.V., Kilin, A.A., and Mamaev, I. S., Dynamics and Control of an Omniwheel Vehicle, Regul. Chaotic Dyn., 2015, vol. 20, no. 2, pp. 153-172.

[11] Dinh, V.-T., Nguyen, H., Shin, S.-M., Kim, H.-K., Kim, S.-B., and Byun, G.-S., Tracking Control of Omnidirectional Mobile Platform with Disturbance Using Differential Sliding Mode Controller, Int. J. Precis. Eng. Manuf., 2012, vol. 13, no. 1, pp. 39-48. 
[12] Huang, H. C. and Tsai, C.C., Adaptive Trajectory Tracking and Stabilization for Omnidirectional Mobile Robot with Dynamic Effect and Uncertainties, IFAC Proc. Vol., 2008, vol. 41, no. 2, pp. 5383-5388.

[13] Huang, H. C. and Tsai, Ch. Ch., Simultaneous Tracking and Stabilization of an Omnidirectional Mobile Robot in Polar Coordinates: A Unified Control Approach, Robotica, 2009, vol. 27, no. 3, pp. 447-458.

[14] Indiveri, G., Paulus, J., and Plöger, P. G., Motion Control of Swedish Wheeled Mobile Robots in the Presence of Actuator Saturation, in RoboCup 2006: Robot Soccer World Cup X, G. Lakemeyer, E. Sklar, D. G. Sorrenti, T. Takahashi (Eds.), Lecture Notes in Comput. Sci., vol. 4434, Berlin: Springer, 2007, pp. 35-46.

[15] Kilin, A., Bozek, P., Karavaev, Yu., Klekovkin, A., and Shestakov, V., Experimental Investigations of a Highly Maneuverable Mobile Omniwheel Robot, Int. J. Adv. Robot. Syst., 2017, vol.14, no. 6, pp. 1-9.

[16] Kilin, A. A. and Karavaev, Yu. L., The Kinematic Control Model for a Spherical Robot with an Unbalanced Internal Omniwheel Platform, Nelin. Dinam., 2014, vol. 10, no.4, pp.497-511 (Russian).

[17] Li, X. and Zell, A., Motion Control of an Omnidirectional Mobile Robot, in Informatics in Control, Automation and Robotics: Selected Papers from the 4th Internat. Conf. on Informatics in Control, Automation and Robotics (Angers, France, 2007): Part 2, J. Filipe, J. A. Cetto, J. L. Ferrier (Eds.), Lecture Notes in Electrical Engineering, vol. 24, Berlin: Springer, 2009, pp. 181-193.

[18] Liu, Y., Zhu, J., Williams, R. L. II, and Wu, J., Omni-Directional Mobile Robot Controller Based on Trajectory Linearization, Robot. Auton. Syst., 2008, vol.56, pp. 461-479.

[19] Martynenko, Yu. G., Stability of Steady Motions of a Mobile Robot with Roller-Carrying Wheels and a Displaced Centre of Mass, J. Appl. Math. Mech., 2010, vol.74, no. 4, pp.436-442; see also: Prikl. Mat. Mekh., 2010, vol. 74, no. 4, pp.610-619.

[20] Martynenko, Yu. G. and Formal'skii, A. M., On the Motion of a Mobile Robot with Roller-Carrying Wheels, J. Comput. Sys. Sc. Int., 2007, vol.46, no.6, pp.976-983; see also: Izv. Ross. Akad. Nauk. Teor. Sist. Upr., 2007, no. 6, pp. 142-149.

[21] Paden, B. and Panja, R., Globally Asymptotically Stable 'PD+' Controller for Robot Manipulators, Int. J. Control, 1988, vol.47, no. 6, pp. 1697-1712.

[22] Pin, F. G. and Killough, S. M., A New Family of Omnidirectional and Holonomic Wheeled Platforms for Mobile Robots, IEEE Trans. Robotics Automat., 1994, vol. 10, no. 2, pp. 480-489.

[23] Purwin, O. and D'Andrea, R., Trajectory Generation and Control for Four Wheeled Omnidirectional Vehicles, Robot. Auton. Syst., 2006, vol. 54, pp. 13-22.

[24] Ren, Ch., Sun, Y., and Ma, Sh., Passivity-Based Control of an Omnidirectional Mobile Robot, Robotics Biomim., 2016, vol.3, no. 10, 9 pp.

[25] Rouche, N., Habets, P., and Laloy, M., Stability Theory by Liapunov's Direct Method, New York: Springer, 1977. Appl. Math. Sci., vol. 22, New York: Springer, 1977.

[26] Santos, J., Conceiçāo, A. G. S., and Santos, T. L. M., Trajectory Tracking of Omni-Directional Mobile Robots via Predictive Control Plus a Filtered Smith Predictor, IFAC PapersOnLine, 2017, vol. 50, no. 1, pp. 10250-10255.

[27] Sell, G. R., Nonautonomous Differential Equations and Topological Dynamics: 2. Limiting Equations, Trans. Amer. Math. Soc., 1967, vol. 127, pp. 263-283.

[28] Vázques, J. A. and Velasco-Villa, M., Path-Tracking Dynamic Model Based Control of an Omnidirectional Mobile Robot, IFAC Proc. Vol., 2008, vol. 41, no. 2, pp. 5365-5373.

[29] Velasco-Villa, M., del-Muro-Cuellary, B., and Alvarez-Aguirre, A., Smith-Predictor Compensator for a Delayed Omnidirectional Mobile Robot, in Proceeding of the 15th Mediterranean Conf. on Control and Automation (Athene, Greece, July 2007), pp. 1-6.

[30] Watanabe, K., Shiraishi, Y., Tzafestas, S. G., Tang, J., and Fukuda, T., Feedback Control of an Omnidirectional Autonomous Platform for Mobile Service Robots, J. Intell. Robot. Syst., 1998, vol. 22, pp. 315-330. 